\title{
Repeatable Noninvasive Measurement of Mouse Myocardial Glucose Uptake with ${ }^{18}$ F-FDG: Evaluation of Tracer Kinetics in a Type 1 Diabetes Model
}

\author{
Stephanie L. Thorn ${ }^{1,2}$, Robert A. deKemp ${ }^{1}$, Tyler Dumouchel ${ }^{1}$, Ran Klein ${ }^{1}$, Jennifer M. Renaud ${ }^{1}$, R. Glenn Wells ${ }^{1}$, \\ Michael H. Gollob ${ }^{2}$, Rob S. Beanlands ${ }^{1,2}$, and Jean N. DaSilva ${ }^{1,2}$ \\ ${ }^{1}$ Division of Cardiology, National Cardiac PET Centre, University of Ottawa Heart Institute, Ottawa, Ontario, Canada; and \\ ${ }^{2}$ Department of Cellular and Molecular Medicine, University of Ottawa, Ottawa, Ontario, Canada
}

\begin{abstract}
A noninvasive and repeatable method for assessing mouse myocardial glucose uptake with ${ }^{18} \mathrm{~F}-\mathrm{FDG}$ PET and Patlak kinetic analysis was systematically assessed using the vena cava imagederived blood input function (IDIF). Methods: Contrast CT and computer modeling was used to determine the vena cava recovery coefficient. Vena cava IDIF $(n=7)$ was compared with the left ventricular cavity IDIF, with blood and liver activity measured ex vivo at $60 \mathrm{~min}$. The test-retest repeatability $(n=9)$ of Patlak influx constant $\mathrm{K}_{\mathrm{i}}$ at 10-40 min was assessed quantitatively using BlandAltman analysis. Myocardial glucose uptake rates (rMGU) using the vena cava IDIF were calculated at baseline $(n=8)$, after induction of type 1 diabetes (streptozotocin [50 mg/kg] intraperitoneally, $5 \mathrm{~d}$ ), and after acute insulin stimulation $(0.08 \mathrm{mU} / \mathrm{kg}$ of body weight intraperitoneally). These changes were analyzed with a standardized uptake value calculation at 20 and 40 min after injection to correlate to the Patlak time interval. Results: The proximal mouse vena cava diameter was $2.54 \pm 0.30 \mathrm{~mm}$. The estimated recovery coefficient, calculated using nonlinear image reconstruction, decreased from 0.76 initially (time 0 to peak activity) to 0.61 for the duration of the scan. There was a $17 \%$ difference in the image-derived vena cava blood activity at $60 \mathrm{~min}$, compared with the ex vivo blood activity measured in the $\gamma$-counter. The coefficient of variability for Patlak $\mathrm{K}_{\mathrm{i}}$ values between mice was found to be $23 \%$ with the proposed method, compared with $51 \%$ when using the left ventricular cavity IDIF $(P<0.05)$. No significant bias in $\mathrm{K}_{\mathrm{i}}$ was found between repeated scans with a coefficient of repeatability of $0.16 \mathrm{~mL} / \mathrm{min} / \mathrm{g}$. Calculated $\mathrm{rMGU}$ values were reduced by $60 \%$ in type 1 diabetic mice from baseline scans $(P<0.03$, ANOVA), with a subsequent increase of $40 \%$ to a level not significantly different from baseline after acute insulin treatment. These results were confirmed with a standardized uptake value measured at 20 and 40 min. Conclusion: The mouse vena cava IDIF provides repeatable assessment of the blood time-activity curve for Patlak kinetic modeling of rMGU. An expected significant reduction in myocardial glucose uptake was demonstrated in a type 1 diabetic mouse model, with significant recovery after acute insulin treatment, using a mouse vena cava IDIF approach.
\end{abstract}

\footnotetext{
Received Jun. 15, 2012; revision accepted Mar. 26, 2013.

For correspondence or reprints contact: Jean N. DaSilva, University of Ottawa Heart Institute, 40 Ruskin St., Ottawa, ON, Canada, K1Y4W7.

E-mail: jdasilva@ottawaheart.ca

Published online Aug. 12, 2013.

COPYRIGHT (C) 2013 by the Society of Nuclear Medicine and Molecular Imaging, Inc.
}

Key Words: positron emission tomography; Patlak; myocardial glucose uptake; streptozotocin; test-retest repeatability

J Nucl Med 2013; 54:1637-1644

DOI: 10.2967/jnumed.112.110114

$\mathbf{M}$ tant target for treatment of cardiovascular diseases, with relatively little information known on the alterations of metabolism in cardiomyopathies (1-3). Transgenic, knockout, pharmacologic, and surgical mouse models are used extensively to study cardiac metabolism. Although mouse models present many positive features-such as the ease in producing customized transgenic or knockout lines and a lower expense in housing fees-the small size of the mouse can present challenging issues.

${ }^{18} \mathrm{~F}-\mathrm{FDG}$ is a glucose analog commonly used in PET to assess cardiac glucose uptake and cell viability (4). ${ }^{18}$ F-FDG enters the cell via glucose transporters ( $K_{1}$ and $k_{2}$ rate constants) and is phosphorylated by hexokinase to ${ }^{18} \mathrm{~F}$-FDG-6-phosphate $\left(k_{3}\right.$ rate constant). The chemical structure of ${ }^{18} \mathrm{~F}-\mathrm{FDG}-6-$ phosphate traps the radiolabeled product within the cell. The ${ }^{18} \mathrm{~F}-\mathrm{FDG}$ uptake rate constant, $\mathrm{K}_{\mathrm{i}}=\left(K_{1} \times k_{3}\right) /\left(k_{2}+k_{3}\right)$, can be estimated using Patlak graphical kinetic analysis, assuming there is negligible tracer washout $\left(k_{4}=0\right)(5) .{ }^{18} \mathrm{~F}$-FDG has been modeled extensively with Patlak analysis in humans $(6,7)$ and rats $(8-12)$. Studies in mice have evaluated the effects of diet (13), anesthetic (14), mode of injection, and blood glucose levels (15) on ${ }^{18}$ F-FDG uptake. However, the inaccuracy of the left ventricular (LV) cavity blood image-derived input function (IDIF) (16-18) remains one of the most difficult impediments to obtaining reliable kinetic measurements in mice. The inaccuracy of the LV cavity IDIF is a particular phenomenon in mice PET imaging because of the relatively small cavity size on the order of the spatial resolution of the current small-animal PET systems ( $\sim 1.2 \mathrm{~mm}$, Inveon DPET; Siemens). Ferl et al. (17) were able to predict a blood input function using modeling by taking 2 arterial samples at approximately 10 and $60 \mathrm{~min}$. Other groups have used serial blood sampling to determine the blood input function $(13,16,19)$. Although manual blood sampling can provide accuracy, it incorporates invasive procedures, reducing serial imaging capabilities. A hybrid image-derived input function has been proposed using the initial peak of the LV cavity IDIF, with the remainder of the curve derived from 
the liver time-activity curve normalized to a 60-min arterial blood sample (20). Although this methodology reported reliable estimates of $\mathrm{K}_{\mathrm{i}}$ using a 2-tissue-compartment model, sampling of arterial blood at $60 \mathrm{~min}$ is still required. Locke et al. (18) recently reported an accurate IDIF with sampling of the LV cavity in mice. However, the accuracy was dependent on using gated iterative reconstruction (12-subset 2-dimensional ordered-subset expectation maximization [OSEM], 2 iterations, 18 maximum a posteriori [MAP] iterations). Although gated OSEM MAP reconstructions allow accuracy at later time frames $(21)$, because of a reduction in spillover from the myocardium, the authors acknowledge that each reconstruction takes approximately $34 \mathrm{~h}$ to complete (18). This methodology presents time-issue constraints in high-output cardiac research centers. Additionally, gated reconstruction relies on accurate electrocardiogram gating that can be compromised in animal models with inherent (22) or induced arrhythmogenesis such as after myocardial ischemic injury. In this study, we describe a novel method that provides a repeatable and accurate cardiac ${ }^{18} \mathrm{~F}-\mathrm{FDG} \mathrm{K}_{\mathrm{i}}$ value using a corrected vena cava IDIF. This approach requires no invasive arterial blood sampling with nongated dynamic iterative reconstruction algorithms. This methodology was tested for repeatability and evaluated in a type 1 diabetic mouse model expected to exhibit decreased ${ }^{18} \mathrm{~F}$-FDG cardiac uptake due to hyperglycemia.

\section{MATERIALS AND METHODS}

\section{Animals}

All animal experiments described herein were conducted according to the guidelines of the University of Ottawa Animal Care Committee and the Canadian Council on Animal Care for the use and care of

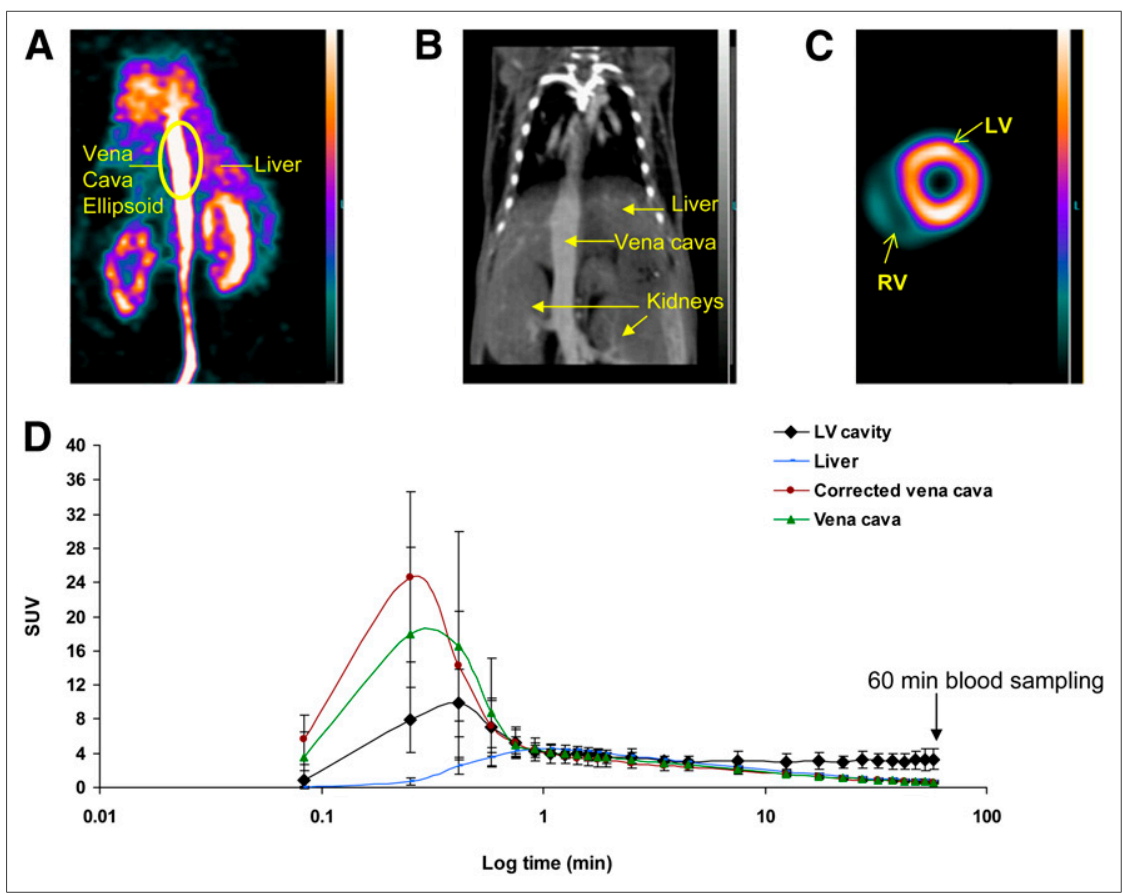

FIGURE 1. Representative images of proximal mouse vena cava and background liver region in early time frames of ${ }^{18} \mathrm{~F}-\mathrm{FDG}$ scan $(A)$, contrast CT of mouse vena cava (B), and mouse ${ }^{18} \mathrm{~F}-\mathrm{FDG}$ heart at $60 \mathrm{~min}(\mathrm{C})$. (D) Time-activity curve displayed in SUVs for 7 mice. Liver, LV cavity, vena cava, and corrected vena cava blood activity curves are displayed over log of time in $60 \mathrm{~min}$. Blood sampling was conducted at 60 -min time point. laboratory animals. All animals were maintained on a 12-h light-dark cycle with chow and water ad libitum. All animals used in this study were male FVB mice (25-34 g) purchased from Charles River Canada. Three groups of mice were used to determine the accuracy of the vena cava IDIF, test-retest repeatability, and ability to measure changes in a type 1 diabetes model. Type 1 diabetes was induced in male FVB mice by streptozotocin, dissolved in a $0.05 \mathrm{M}$ sodiumcitrate solution, injected at $50 \mathrm{mg} / \mathrm{kg}$ intraperitoneally once daily for $5 \mathrm{~d}$ (23). Mice were fasted $4-6 \mathrm{~h}$ before streptozotocin injection to increase conversion. At $10 \mathrm{~d}$ after streptozotocin injection, fasting blood glucose levels (2-3 h fast) were measured as described in the "Blood Markers" section. Mice with levels higher than $15 \mathrm{mmol} / \mathrm{L}$ were categorized as having type 1 diabetes.

\section{Reconstructed Image Resolution}

Measurement of the partial-volume effect in the vena cava will depend on the size of the vessel and scanner resolution. We therefore conducted experiments to determine the recovery coefficient (RC) for the mouse vena cava. The size of the vena cava was determined in 3 mice using CT contrast imaging (nanoSPECT/CT; BioScan). Briefly, animals were anesthetized with isoflurane ( $2 \%$ isoflurane, $2 \mathrm{~mL} / \mathrm{min}$ oxygen) and injected intravenously with $0.2 \mathrm{~mL}$ of Exia CT contrast (SKYSCAN). A 30-s topogram was completed immediately after injection to select the field of view (FOV) over the proximal vena cava. A 9-min CT scan was reconstructed $(45 \mathrm{kVp}, 1,500 \mathrm{~ms}$ exposure time, 360 projections) with $0.2-\mathrm{mm}$ transaxial pixel size. The proximal vena cava diameter located above the kidneys and below the myocardium was measured by an average of 3 measurements on each of the coronal, sagittal, and axial planes (IRW software; Siemens). We subsequently determined the recovery coefficient on the Inveon PET scanner for the vena cava using the full width at half maximum (FWHM) of the gaussian point spread function (PSF) scanner resolution with the perturbation technique (24). Briefly, a ${ }^{22} \mathrm{Na}$ point source (diameter, $0.25 \mathrm{~mm} ; 1 \mathrm{kBq}$ ) embedded in a 1$\mathrm{cm}^{3}$ acrylic cube was placed within the PET scanner such that the point source location corresponded to the center of the vena cava on a reconstructed transaxial PET image. A 5-min acquisition was performed and the data binned into 3-dimensional sinograms. The high-count-density point source sinograms were scaled and added to the mouse sinogram data and reconstructed. The point source sinogram scaling ensures that only a small perturbation is introduced (i.e., $<1 \%$ of the counts in each mouse sinogram bin) so that the convergence properties of the reconstructed image are minimally affected. The perturbed images were reconstructed using the same parameters as the original mouse images, and the difference was computed to obtain the PSF. The reconstructed image FWHM resolution was determined by fitting a gaussian function to the PSF difference image.

\section{Small-Animal PET Imaging}

Small-animal PET ${ }^{18} \mathrm{~F}-\mathrm{FDG}$ imaging was conducted with the Inveon DPET small-animal scanner. A 60-min list-mode acquisition, together with a 10- to 20-s tail vein injection of ${ }^{18} \mathrm{~F}-\mathrm{FDG}(7-82 \mathrm{MBq}$ in $150 \mu \mathrm{L})$, was started. List data were sorted into 26 dynamic frames 
TABLE 1

Image-Derived Activity, Compared with $\gamma$-Counter Activity, at 60 Minutes

\begin{tabular}{|c|c|c|c|c|c|c|}
\hline \multirow[b]{2}{*}{ Mouse no. } & \multicolumn{2}{|c|}{ Liver activity $\left(\mathrm{kBq} / \mathrm{cm}^{3}\right)$} & \multicolumn{4}{|c|}{ Blood activity $\left(\mathrm{kBq} / \mathrm{cm}^{3}\right)$} \\
\hline & Image-derived & $\gamma$-counter & LV cavity & Vena cava & Vena cava-to-liver ratio & $\gamma$-counter \\
\hline 1 & 952 & 1005 & 3,366 & 811 & 750 & 589 \\
\hline 2 & 907 & 801 & 2,097 & 585 & 489 & 442 \\
\hline 3 & 787 & 647 & 2,095 & 539 & 432 & 316 \\
\hline 4 & 833 & 711 & 2,484 & 566 & 483 & 391 \\
\hline 5 & 974 & 651 & 2,875 & 507 & 366 & 557 \\
\hline 6 & 866 & 637 & 3,802 & 431 & 371 & 260 \\
\hline 7 & 1018 & 845 & 3,055 & 612 & 540 & 373 \\
\hline Mean & 905 & 757 & 2,825 & 579 & 490 & 418 \\
\hline $\mathrm{SD}$ & 82 & 136 & 643 & 118 & 131 & 121 \\
\hline Coefficient of variation & 0.09 & 0.18 & 0.23 & 0.20 & 0.27 & 0.29 \\
\hline Bias to $\gamma$-counter & $20 \%$ * & & $575 \%$ * & $38 \% *$ & $17 \%$ & \\
\hline
\end{tabular}

$(12 \times 10 \mathrm{~s}, 3 \times 60 \mathrm{~s}$, and $11 \times 300 \mathrm{~s})$ and reconstructed using 3-dimensional OSEM with 10 iterations, 16 subsets, and a zoom of 2.5 with a $128 \times 128$ matrix, resulting in 0.35 -mm transaxial pixel size. Images were corrected for radioactive decay, random coincidences, and deadtime losses using the vendor software (IAW, version 1.5; Siemens).

The average vena cava activity was determined from a region of interest (ROI) placed in the corresponding region of the PET image and thresholded to $50 \%$ of the local maximum vena cava activity $\left(V C_{50}\right)$. The ROI was positioned manually above the kidneys and below the myocardial blood pool in the initial time frames, where the injected tracer bolus is clearly visible.

The RC for the measured vena cava activity was estimated by convolving a circular model of the vena cava with measured diameter, together with the 2-dimensional gaussian PSF representing the reconstructed PET image resolution. Likewise, a complementary uniform background activity was convolved with the same PSF to represent spill-in of activity from the liver into the vena cava ROI. An estimate of the true vena cava activity $\left(A_{V C}\right)$ can then be obtained using the following relationship:

$$
A_{V C}=\frac{V C_{50}-\left(1-R C_{50}\right) B}{R C_{50}}
$$

where $B$ is the background activity determined by placing an ROI adjacent to the vena cava. The adjacent background used for this ROI is the liver (Fig. 1A), which typically has homogeneous ${ }^{18} \mathrm{~F}-$ FDG activity uptake and is the largest feature proximal to the vena cava.

Myocardial images were analyzed and quantified with Patlak kinetic analysis $\left(\mathrm{K}_{\mathrm{i}}\right)$ at 10-40 $\mathrm{min}$ from the reconstructed images using FlowQuant semiautomated software (University of Ottawa Heart Institute [UOHI]) (25-27). In brief, uptake images of the left ventricle were formed using the last $5 \mathrm{~min}$ of scan data. The location, orientation, and size of the left ventricle were automatically determined by fitting ellipses to the myocardium in the transverse, vertical long-axis, and horizontal long-axis planes. Transverse uptake images were reoriented automatically into short-axis sections, generating LV slices from the apex to the base plane. Polar maps of the relative uptake activity (\%) were formed from the sampled data. Blood ROIs were automatically placed in the LV cavity. The sampling points were then applied to all time frames to generate myocardial and blood time-activity curves. Patlak kinetic analysis was applied to the myocardial data using either the blood input function from the FlowQuant automated cavity measurement or the estimated vena cava blood input function (Eq. 1) imported into FlowQuant. This was done such that the exact same myocardium orientations and LV sampling were used with both blood input functions.

Regional rMGU was determined for each animal using the following standard equation:

$$
\mathrm{rMGU}=\mathrm{K}_{\mathrm{i}} \times \mathrm{BG} / \mathrm{LC}(\mathrm{mmol} / \mathrm{min} / \mathrm{g}),
$$

where $\mathrm{K}_{\mathrm{i}}$ is the Patlak uptake rate constant, BG is the blood glucose concentration $(\mathrm{mmol} / \mathrm{mL})$ obtained before ${ }^{18} \mathrm{~F}-\mathrm{FDG}$ injection, and LC is the lumped constant equal to 0.67 , which accounts for differences in the uptake and phosphorylation of ${ }^{18} \mathrm{~F}-\mathrm{FDG}$ versus glucose.

${ }^{18} \mathrm{~F}$-FDG uptake in the type 1 diabetic group was also quantified using a standardized uptake value (SUV) at 20 and 40 min to independently evaluate myocardial radiotracer uptake. SUV was calculated according to the following standard equation:

$$
\mathrm{SUV}=\frac{\text { Activity concentration in a region of interest }\left(\mathrm{Bq} / \mathrm{cm}^{3}\right)}{\text { Injected activity }(\mathrm{Bq}) / \text { weight of the animal }(\mathrm{g})},
$$

\section{Image-Derived Input Function Accuracy}

To determine the accuracy of the calculated IDIF, 7 mice were scanned after intravenous injection of ${ }^{18} \mathrm{~F}-\mathrm{FDG}$, with measurements of blood activity at $60 \mathrm{~min}$, compared with a manual blood sample obtained from trunk blood after decapitation after the scan. Liver was also collected as a homogeneous tissue region. ${ }^{18} \mathrm{~F}-\mathrm{FDG}$ activity of the samples was measured with a Packard Cobra II $\gamma$-counter (Perkin Elmer) and expressed as percentage injected dose (activity) per gram of tissue $(\% \mathrm{ID} / \mathrm{g})$. \% ID/g of each sample was multiplied by the injected activity and divided by $100 \%$ to obtain the activity per cubic centimeter of tissue $\left(\mathrm{Bq} / \mathrm{cm}^{3}\right)$, assuming a tissue density of $1 \mathrm{~g} / \mathrm{cm}^{3}$.

\section{Test-Retest Repeatability}

To evaluate the repeatability of Patlak $\mathrm{K}_{\mathrm{i}}$ measurements using the calculated IDIF, 9 mice were scanned twice $3 \mathrm{~d}$ apart, using the same procedures listed above. Blood glucose levels were sampled immediately before ${ }^{18} \mathrm{~F}$-FDG injection. An additional blood sample was acquired to measure insulin and free fatty acid (FFA) levels. Patlak 


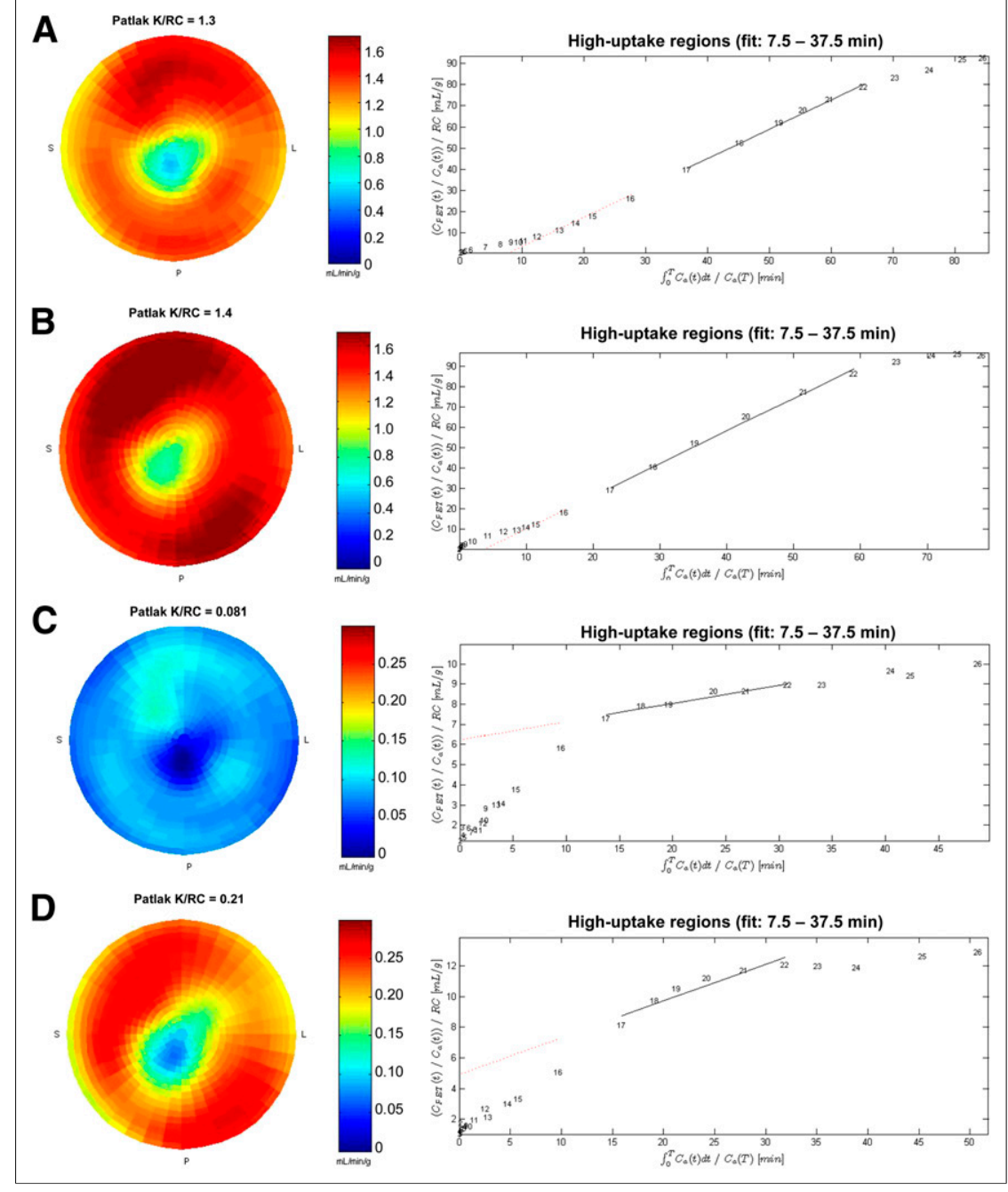

FIGURE 2. Patlak polar maps and plots of representative test-retest mouse using vena cava blood input function for scan 1 (A) and scan 2 (B) and using LV cavity blood input function for scan 1 (C) and scan 2 (D).

$\mathrm{K}_{\mathrm{i}}$ values were compared with calculated 2-compartment model $\mathrm{K}_{\mathrm{i}}$ values and SUV measurements.

\section{Type I Diabetes}

Mice $(n=8)$ were scanned with ${ }^{18} \mathrm{~F}-\mathrm{FDG}$ at baseline and $2 \mathrm{wk}$ after streptozotocin injection. An additional scan was conducted 3-4 $\mathrm{d}$ later with short-acting insulin $(0.08 \mathrm{mU} / \mathrm{kg}$ of body weight intraperitoneally; Novolin ge [Novo Nordisk]) injected $30 \mathrm{~min}$ before the ${ }^{18}$ F-FDG scan to assess insulin sensitivity (28). Blood glucose levels for all scans were measured immediately before ${ }^{18} \mathrm{~F}$-FDG injection, with an additional blood sample acquired for measuring insulin and FFA levels.

\section{Blood Markers}

Blood was obtained from the saphenous vein with a 25 -gauge needle by shaving the back of the leg and applying an alcohol wipe and petroleum jelly to help pool the blood. Blood glucose concentration was measured $(\mathrm{mmol} / \mathrm{L})$ with a small drop of blood using the Advantage strips (AccuChek; Roche Diagnostics). Plasma insulin concentrations were measured with the Mouse Ultrasensitive Insulin ELISA kit (ALPCO Diagnostics). Results were analyzed using the GraphPad Prism software (GraphPad Software, Inc.) and expressed in
ng/mL. The plasma FFAs were measured with the commercially available Free Fatty Acid Quantification Kit (BioVision Inc.), with concentration of each sample expressed as $\mathrm{mmol} / \mathrm{L}$.

\section{Statistical Analysis}

Curve fitting with FlowQuant software was performed using MATLAB (The MathWorks). All data are expressed as mean \pm SD. Data were compared using either an $F$ test for variance, a $\mathrm{Z}$ test, a Student $t$ test, or a 1-way ANOVA with multiple comparisons between groups using a Bonferroni post hoc comparison. A $P$ value of less than 0.05 was considered significant.

\section{RESULTS}

\section{Vena Cava Measurements}

The measured average diameter of the mouse vena cava was $2.54 \pm 0.30 \mathrm{~mm}$ (Fig. 1B). The image FWHM resolution was estimated as $1.02 \pm 0.04 \mathrm{~mm}$ in the early frames (from time 0 to the peak vena cava activity), compared with $1.71 \pm$ $0.12 \mathrm{~mm}$ in the subsequent time frames. This difference in image resolution is due to the known nonlinear effects of OSEM image reconstruction that are dependent on temporal changes in local count-density and contrast. Using this time-dependent image PSF and the circular vessel dimension from $\mathrm{CT}$, the vena cava $\mathrm{RC}$ value of $0.76 \pm 0.04$ estimated for the early frames was significantly higher than $0.61 \pm 0.07$ used for later time frames following the peak vena cava activity.

\section{IDIF and Organ Activity Accuracy}

The transaxial FOV on the Inveon DPET scanner is $10 \mathrm{~cm}$, and the axial FOV is $12.8 \mathrm{~cm}$, allowing for the whole mouse to be imaged in a single bed position. The left ventricle, when placed in the center of the FOV, has excellent resolution and contrast. The right ventricle is also apparent (Fig. 1C). Table 1 presents the image-derived activities from the liver, vena cava, and LV cavity, compared with the tissue activities measured with the $\gamma$-counter. Evident in the liver, where there is relative homogeneity of signal and minimal partial-volume effect due to the large size of the organ, there is a slight bias in measurements (20\%) between the PET image and the $\gamma$-counter. The blood activity from both the LV cavity and the vena cava had lower population variability, compared with $\gamma$-counter measurements of the trunk blood ( $23 \%$ and $20 \%$ vs. $29 \%$, respectively; $P<0.01, \mathrm{~F}_{8}$ ). The LV cavity blood activity measured from the PET images at 60 min was almost 6-fold higher than the measured $\gamma$-counter activity, whereas the vena cava PET image activity was only $38 \%$ higher. When the vena cava activity was normalized using the image-to- $\gamma$-counter activity ratio in the liver (liver ratio), this difference was further reduced to $17 \%$. The full 60 -min blood input functions ( $n=7$ mice) were compared for the LV 


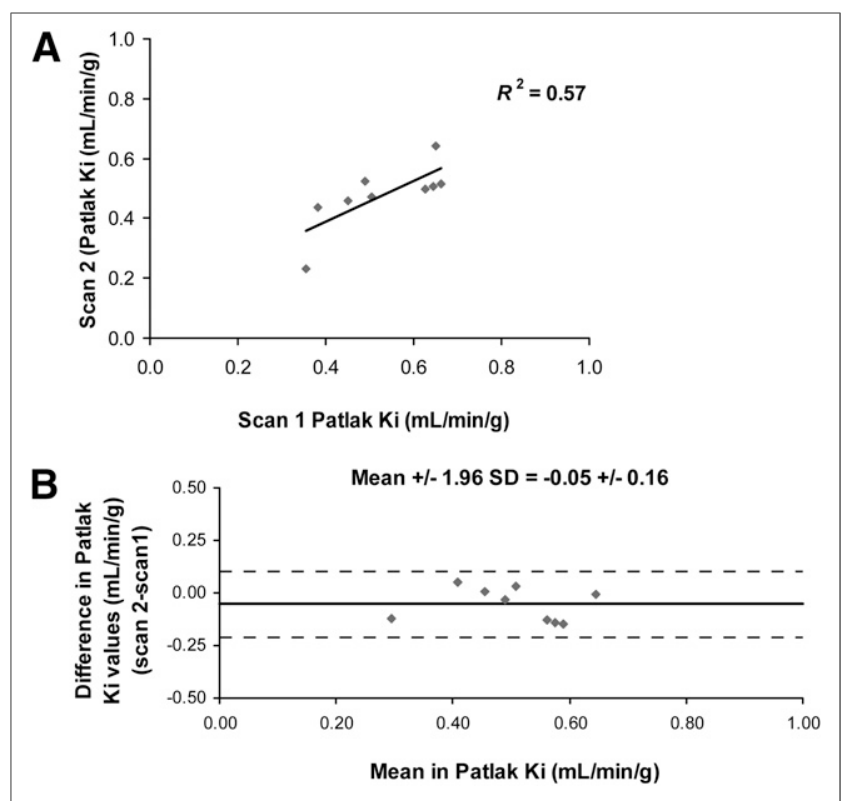

FIGURE 3. Comparison of test-retest Patlak $K_{i}$ values using vena cava IDIF (A), with dashed line representing line of identity. BlandAltman plots of test-retest Patlak K using vena cava IDIF (B).

cavity, uncorrected vena cava, liver (background region), and calculated vena cava activity (Fig. 1D). The early peak of the vena cava input function was higher than the LV cavity curve and demonstrated a slow decline in the later time frames, as expected, whereas the LV cavity curve actually displayed a slight increase, potentially because of activity spilling in from the surrounding myocardium.

\section{Test-Retest Repeatability}

Because there were only $3 \mathrm{~d}$ between scans, there was no difference in body weight. Blood glucose and insulin values were not significantly different between scans; however, plasma FFA was significantly decreased, approximately $41 \%$ (47.9 \pm 11.1 vs. $28.0 \pm 5.4 \mathrm{mmol} / \mathrm{L}, P<0.05, t$ test).

Figure 2 shows representative polar maps and Patlak analysis curves from 1 mouse. Figures $2 \mathrm{~A}$ and $2 \mathrm{~B}$ are test-retest results in mouse 1 using the corrected vena cava blood input function. Patlak polar maps appear similar, with an excellent linear fit observed at the specified interval of 10-40 min. Patlak $\mathrm{K}_{\mathrm{i}}$ values had a range of 0.23 to $0.66 \mathrm{~mL} / \mathrm{min} / \mathrm{g}$ between mice, with a mean of $0.50 \pm$ $0.11 \mathrm{~mL} / \mathrm{min} / \mathrm{g}$ representing a $23 \%$ population coefficient of variability and good repeatability (Fig. $3 \mathrm{~A}, r^{2}=0.57$ ). Figures $2 \mathrm{C}$ and $2 \mathrm{D}$ show the same mouse test-retest scans using the LV cavity as the blood input function. There is a clear difference in the Patlak polar maps and a relatively poor fit to the data, with $\mathrm{K}_{\mathrm{i}}$ values ranging from 0.08 to $0.37 \mathrm{~mL} / \mathrm{min} / \mathrm{g}$ between mice and a coefficient of variability of $51 \%$ (mean, $0.15 \pm 0.08 \mathrm{~mL} / \mathrm{min} / \mathrm{g}$ ).
Bland-Altman plots of the Patlak $\mathrm{K}_{\mathrm{i}}$ values from scans 1 and 2 using the corrected vena cava blood input function had no significant bias $(P>0.05, t$ test $)$ (Fig. 3B) and a coefficient of repeatability of $0.16 \mathrm{~mL} / \mathrm{min} / \mathrm{g}$ ( $32 \%$ of mean). We compared these results to $\mathrm{K}_{\mathrm{i}}$ values derived from a 2-comparment $3 \mathrm{~K}$ model and SUV at 30- to 60-min frames (Table 2). We found that the 2compartment model had a higher population variability at $31 \%$ (compared with $23 \%$ with Patlak $\mathrm{K}_{\mathrm{i}}$ ) and a higher coefficient of repeatability at $39 \%$ (compared with $32 \%$ with Patlak). SUV analysis provides the lowest values, with $21 \%$ population variability and a coefficient of repeatability of $17 \%$.

\section{Type 1 Diabetes}

Body weight and plasma insulin were significantly reduced in the type 1 diabetic state, compared with baseline values $(P<0.05$, ANOVA Bonferroni) (Table 3). Plasma FFA levels were increased significantly only during the acute insulin scan. As expected, blood glucose levels were significantly increased in the type 1 diabetic state and reduced to baseline levels after acute insulin stimulation before ${ }^{18}$ F-FDG injection (Table 3). Representative images display an excellent fit using Patlak analysis with the corrected vena cava blood input at baseline (Fig. 4A), during untreated type 1 diabetes (Fig. 4B), and after acute treatment with insulin (Fig. 4C). rMGU values were significantly reduced by $60 \%$ from baseline to type 1 diabetes. After acute insulin treatment, rMGU increased by $40 \%$ toward baseline values (Table 4 ). Interpretation of these changes was confirmed with similar relative changes in SUV at 20 and $40 \mathrm{~min}$ (Table 4).

\section{DISCUSSION}

In this study, we developed a methodology for acquiring an accurate and repeatable blood IDIF using the vena cava in mice. Using a fed state with isoflurane as the anesthetic, we found as in previous studies $(13-15,17,18)$ that the myocardium had high tissue-to-background contrast. However, the small size of the mouse LV cavity on the order of the spatial resolution of the small-animal PET scanner, with substantial myocardial activity spillover, resulted in an inaccurate assessment of the blood IDIF. Although an SUV can be used to compare activity concentration in a tissue of interest at 1 particular time point, it has been noted that kinetic modeling is needed to assess even the simplest of changes in metabolic rate constants (13), such as increased ${ }^{18} \mathrm{~F}-\mathrm{FDG}$ uptake rate with insulin stimulation.

We investigated the proximal portion of the vena cava for measurement of an IDIF. In human studies, venous and arterial concentrations of ${ }^{18} \mathrm{~F}-\mathrm{FDG}$ are considered to be equal at $30-$ 60 min after injection of tracer (29). In mice, with their high cardiac output rate, we hypothesized that the distribution of tracer from venous to arterial blood occurs at a substantially higher rate. Additionally, the location of the vena cava for ${ }^{18} \mathrm{~F}-\mathrm{FDG}$ scans removes most high-activity areas from the proximity of the vena cava ROI, reducing activity spillover from adjacent background

TABLE 2

Comparison of Kinetic Modeling Measurements

\begin{tabular}{lccc}
\hline \multicolumn{1}{c}{ Descriptive } & Patlak $\mathrm{K}_{\mathrm{i}}$ & 2-compartment 3K model $\mathrm{K}_{\mathrm{i}}$ & SUV (30-60 min) \\
\hline Mean \pm SD & $0.50 \pm 0.1$ & $0.37 \pm 0.1$ & $15.46 \pm 3.3$ \\
Population variability (\%) & 23 & 31 & 21 \\
Coefficient of repeatability (\%) & 32 & 39 & 17 \\
\hline
\end{tabular}


TABLE 3

Body Weight and Blood Markers in Type 1 Diabetes Mellitus Mice

\begin{tabular}{lcccc}
\hline \multicolumn{1}{c}{ Group } & Body weight $(\mathrm{g})$ & Blood glucose $(\mathrm{mmol} / \mathrm{L})$ & Insulin $(\mathrm{ng} / \mathrm{mL})$ & $\mathrm{FFA}(\mathrm{mmol} / \mathrm{L})$ \\
\hline Controls & $29.7 \pm 1.8$ & $11.3 \pm 3.3$ & $0.93 \pm 0.32$ & $40.2 \pm 6.8$ \\
Type 1 DM & $24.9 \pm 1.5$ & $22.6 \pm 7.6^{\star}$ & $0.08 \pm 0.03^{*}$ & $65.4 \pm 14.4$ \\
Type 1 DM + insulin & $25.2 \pm 1.4$ & $12.5 \pm 6.2$ & $0.18 \pm 0.05^{*}$ & $92.1 \pm 14.2^{*}$ \\
& & & & \\
*1-way ANOVA, Bonferroni $P$ value $<0.05$. & & & \\
DM = diabetes mellitus.
\end{tabular}

regions. The adjacent background used for this ROI is the liver, which typically has homogeneous ${ }^{18} \mathrm{~F}$-FDG activity uptake. We were able to derive an RC for the vena cava using measured values of the reconstructed image resolution to estimate the true vena cava activity (Eq. 1). Manual blood sampling was performed at 60 min to confirm the accuracy of the calculated vena cava blood activity. We found a $17 \%$ difference between the measured blood activity and the image-derived vena cava activity when using the liver as a standard between the small-animal PET camera and $\gamma$-counter. This difference was substantially lower than the blood input function derived from the LV cavity, which was approximately $575 \%$ higher than the $\gamma$-counter-measured blood activity. To compare with previously published data from Locke et al. (18), we reconstructed our data using their reconstruction parameters without gating. Despite an improvement from our calculated values, the IDIF from the LV cavity was $130 \%$ different from mea-

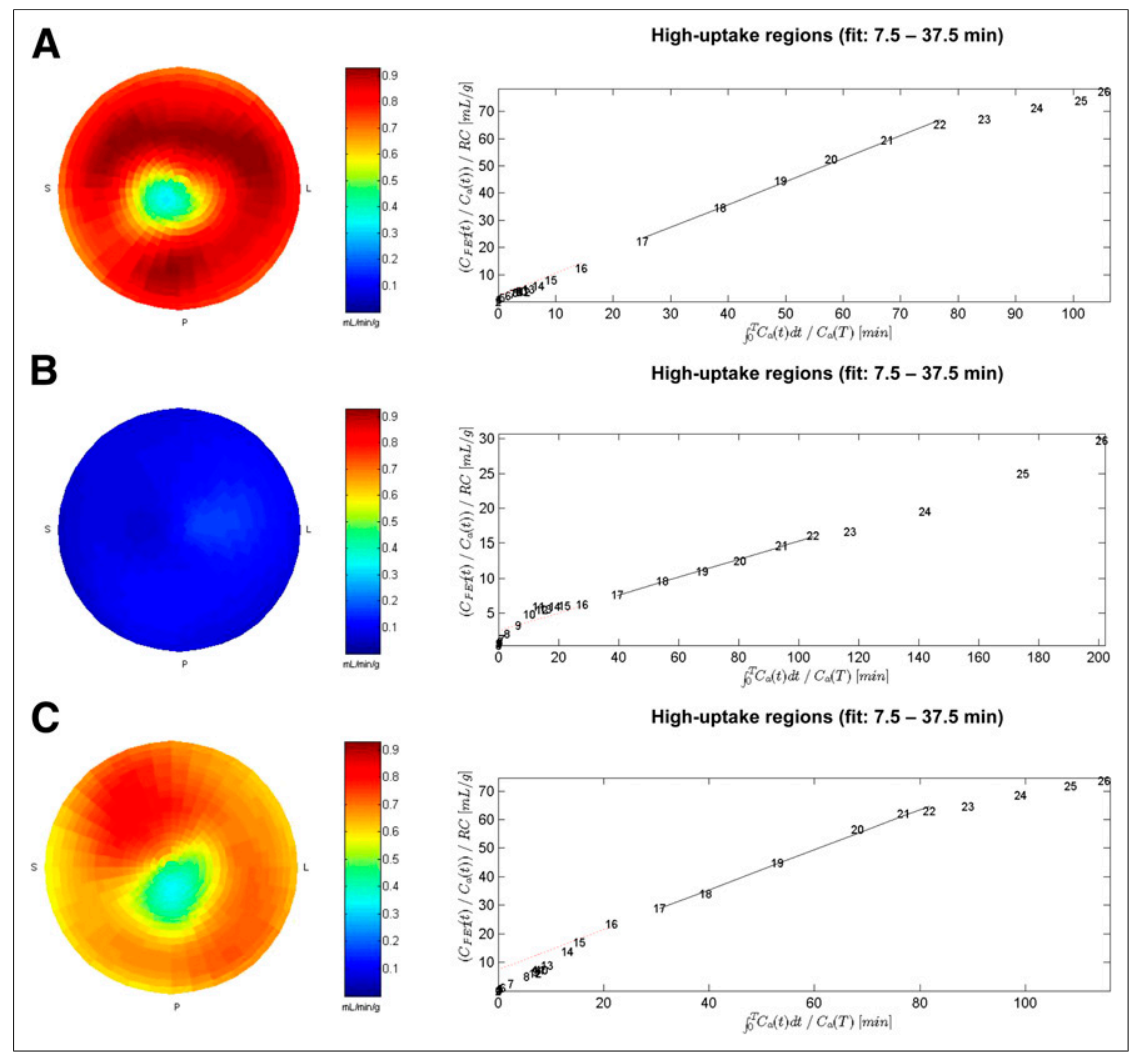

FIGURE 4. Representative Patlak polar maps and plots at baseline (A), after type 1 diabetes induction (B), and after acute insulin stimulation (C). sured $\gamma$-counter activity. As stated in their paper, gating to reduce activity spillover from the myocardium is essential to determine an accurate LV cavity IDIF (18). However, long reconstruction times ( $\sim 34 \mathrm{~h}$ per mouse) and dependency on accurate electrocardiogram gating in all animal models render this method difficult to execute.

The vena cava time-activity curves display an earlier, sharper, and higher initial input function than the LV cavity curves. We postulate that this does not adversely affect the accuracy of the Patlak analysis because integral activity is used for the analysis, and the shape of the curve outside the analysis time range (10-40 $\mathrm{min})$ is of no consequence. Later time frames with the vena cava IDIF display a slow decline in activity concentration, consistent with ${ }^{18} \mathrm{~F}-\mathrm{FDG}$ uptake and retention in tissue, rather than the increase in activity concentration observed with the LV cavity. We presume that the increase in the LV cavity blood input function is due to myocardial spillover. Kreissl et al. (13) noted that the initial time frames when the left ventricle had low myocardial ${ }^{18} \mathrm{~F}$-FDG uptake were appropriate for measurements of the LV cavity blood input function, whereas later frames of the blood IDIF can be derived through computer modeling with 2 blood samples.

The Patlak $\mathrm{K}_{\mathrm{i}}$ test-retest population variability was $23 \%$ with the vena cava blood input function, reduced from $51 \%$ when using the LV cavity blood input function $(P<0.05)$. The Patlak $\mathrm{K}_{\mathrm{i}}$ coefficient of repeatability with the vena cava blood input function was $32 \%$, with no significant bias between scan 1 and scan $2(P>0.05)$. We additionally evaluated $K_{i}$ for the 2compartment model and found an increase in both the test-retest population variability and the coefficient of repeatability, which could be explained by the larger number of free parameters in the model.

We validated this methodology in a streptozotocin-induced type 1 diabetic mouse model. Type 1 diabetes is known to exhibit a reduction in insulin-stimulated glucose uptake due to an overall reduction in endogenous insulin. Although the type 1 diabetes model will produce large perturbations in rMGU values, we chose this model because it is a well-established model of hyperglycemia and alterations in myocardial glucose uptake. Induction of 
TABLE 4

rMGU and SUVs at Baseline, After Induction of Type 1 Diabetes, and After Acute Insulin Treatment

\begin{tabular}{lclc}
\hline Measurement & Baseline & Type 1 DM & Type 1 DM + insulin \\
\hline rMGU & $18.9 \pm 6.4$ & $7.7 \pm 3.7^{*}$ & $12.8 \pm 6.0$ \\
SUV & & & \\
$20 \mathrm{~min}$ & $10.8 \pm 2.9$ & $2.9 \pm 0.9^{*}$ & $7.7 \pm 3.9$ \\
$40 \mathrm{~min}$ & $13.3 \pm 3.8$ & $3.6 \pm 1.3^{*}$ & $8.3 \pm 3.6$ \\
\\
\end{tabular}

type 1 diabetes significantly reduced rMGU by $60 \%$, compared with baseline scans $(P<0.05)$. We further validated that we could measure an increased glucose uptake with an acute treatment of insulin in the type 1 diabetic mouse. A significant $40 \%$ increase in rMGU was observed, returning the measurement close to baseline prediabetic levels $(P>0.05)$. These changes were supported by SUVs calculated at 20 and $40 \mathrm{~min}$, capturing 2 time frames within the Patlak $\mathrm{K}_{\mathrm{i}}$ time interval of 10-40 min.

In clinical studies, patients fast for a minimum of $6 \mathrm{~h}$ before scanning, with nondiabetic patients receiving an oral glucose load before ${ }^{18} \mathrm{~F}$-FDG injection and diabetic patients an insulineuglycemic clamp to ensure that baseline blood glucose levels are not significantly different (30-32). Kreissl et al. (13) found that as in humans, fasting reduced cardiac ${ }^{18} \mathrm{~F}-\mathrm{FDG}$ uptake rates in mice by approximately 5- to 6-fold. We did not perform our methodology under fasting conditions with an insulin-euglycemic clamp because the objective was to characterize the changes (if any) in the type 1 diabetic model from baseline, and the use of a clamp would normalize diabetic glucose uptake, as has been previously reported in human patients (33). All ${ }^{18} \mathrm{~F}$-FDG imaging protocols were conducted in the fed state.

One of our study's limitations is the fact that blood sampling for accuracy was performed only at the 60-min time point as opposed to sampling throughout the scan. Although the authors acknowledge this as a limitation, the 60 -min time point is representative of when the highest myocardial spillover is present and contaminating the LV cavity blood input curve. This method is also strongly dependent on the RC values, which may change with alterations in the reconstruction parameters, both postreconstruction filters and MAP priors, number of iterations, and sizes of subsets. As such, the results are applicable only to the parameters used in this study. The degree that spillover influences the measured values depends heavily on the distribution of the tracer background uptake. Extending this approach to other tracers or using this in models for which the uptake of ${ }^{18} \mathrm{~F}$-FDG in the liver or blood clearance altered should be approached with caution and may require additional validation.

\section{CONCLUSION}

A new methodology for measuring mouse ${ }^{18} \mathrm{~F}-\mathrm{FDG}$ myocardial glucose uptake was developed using an image-derived blood input function with excellent repeatability. Additionally, this is the first study, to our knowledge, to demonstrate alterations in rMGU measurements of ${ }^{18} \mathrm{~F}$-FDG in a mouse type 1 diabetic model. This methodology allows users to quantify changes in rMGU serially and noninvasively in mouse models with ${ }^{18} \mathrm{~F}$-FDG and further translate this information directly to the clinical setting.

\section{DISCLOSURE}

The costs of publication of this article were defrayed in part by the payment of page charges. Therefore, and solely to indicate this fact, this article is hereby marked "advertisement" in accordance with 18 USC section 1734 . This study was supported in part by the Heart and Stroke Foundation of Ontario (HSFO) Program Grant on Molecular Function and Imaging (no. PRG6242) and a Heart and Stroke Foundation PhD Scholarship. No other potential conflict of interest relevant to this article was reported.

\section{ACKNOWLEDGMENTS}

We thank Julia Lockwood for technical assistance with CT imaging and the Animal Care and Veterinary services of the University of Ottawa for their assistance with animal health.

\section{REFERENCES}

1. Horowitz JD, Chirkov YY, Kennedy JA, Sverdlov AL. Modulation of myocardial metabolism: an emerging therapeutic principle. Curr Opin Cardiol. 2010;25: 329-334.

2. Wang W, Lopaschuk GD. Metabolic therapy for the treatment of ischemic heart disease: reality and expectations. Expert Rev Cardiovasc Ther. 2007;5:11231134 .

3. Lionetti V, Stanley WC, Recchia FA. Modulating fatty acid oxidation in heart failure. Cardiovasc Res. 2011;90:202-209.

4. Yoshinaga K, Chow BJ, deKemp R, et al. Application of cardiac molecular imaging using positron emission tomography in evaluation of drug and therapeutics for cardiovascular disorders. Curr Pharm Des. 2005;11:903-932.

5. Patlak CS, Blasberg RG, Fenstermacher JD. Graphical evaluation of blood-tobrain transfer constants from multiple-time uptake data. J Cereb Blood Flow Metab. 1983;3:1-7.

6. Abraham A, Nichol G, Williams KA, et al. ${ }^{18}$ F-FDG PET imaging of myocardial viability in an experienced center with access to ${ }^{18} \mathrm{~F}-\mathrm{FDG}$ and integration with clinical management teams: the Ottawa-FIVE substudy of the PARR 2 trial. J Nucl Med. 2010;51:567-574.

7. Ha AC, Renaud JM, Dekemp RA, et al. In vivo assessment of myocardial glucose uptake by positron emission tomography in adults with the PRKAG2 cardiac syndrome. Circ Cardiovasc Imaging. 2009;2:485-491.

8. Shoghi KI, Finck BN, Schechtman KB, et al. In vivo metabolic phenotyping of myocardial substrate metabolism in rodents: differential efficacy of metformin and rosiglitazone monotherapy. Circ Cardiovasc Imaging. 2009;2:373-381.

9. Shoghi KI, Gropler RJ, Sharp T, et al. Time course of alterations in myocardial glucose utilization in the Zucker diabetic fatty rat with correlation to gene expression of glucose transporters: a small-animal PET investigation. $J$ Nucl Med. 2008;49:1320-1327.

10. Herrero P, Dence CS, Sharp TL, Welch MJ, Gropler RJ. Impact of reversible trapping of tracer and the presence of blood metabolites on measurements of myocardial glucose utilization performed by PET and ${ }^{18} \mathrm{~F}$-fluorodeoxyglucose using the Patlak method. Nucl Med Biol. 2004;31:883-892.

11. Ménard SL, Croteau E, Sarrhini O, et al. Abnormal in vivo myocardial energy substrate uptake in diet-induced type 2 diabetic cardiomyopathy in rats. Am J Physiol Endocrinol Metab. 2010;298:E1049-E1057.

12. Ménard SL, Ci X, Frisch F, et al. Mechanism of reduced myocardial glucose utilization during acute hypertriglyceridemia in rats. Mol Imaging Biol. 2009;11:6-14.

13. Kreissl MC, Stout DB, Wong KP, et al. Influence of dietary state and insulin on myocardial, skeletal muscle and brain [F]-fluorodeoxyglucose kinetics in mice. EJNMMI Res. 2011;1:8.

14. Flores JE, McFarland LM, Vanderbilt A, Ogasawara AK, Williams SP. The effects of anesthetic agent and carrier gas on blood glucose and tissue uptake in mice undergoing dynamic FDG-PET imaging: sevoflurane and isoflurane compared in air and in oxygen. Mol Imaging Biol. 2008;10:192-200.

15. Wong KP, Sha W, Zhang X, Huang SC. Effects of administration route, dietary condition, and blood glucose level on kinetics and uptake of ${ }^{18} \mathrm{~F}-\mathrm{FDG}$ in mice. J Nucl Med. 2011;52:800-807.

16. Fang YH, Muzic RF Jr. Spillover and partial-volume correction for imagederived input functions for small-animal ${ }^{18}$ F-FDG PET studies. J Nucl Med. 2008;49:606-614. 
17. Ferl GZ, Zhang X, Wu HM, Kreissl MC, Huang SC. Estimation of the ${ }^{18}$ F-FDG input function in mice by use of dynamic small-animal PET and minimal blood sample data. J Nucl Med. 2007;48:2037-2045.

18. Locke LW, Berr SS, Kundu BK. Image-derived input function from cardiac gated maximum a posteriori reconstructed PET images in mice. Mol Imaging Biol. 2011;13:342-347.

19. Convert L, Morin-Brassard G, Cadorette J, Archambault M, Bentourkia M, Lecomte R. A new tool for molecular imaging: the microvolumetric beta blood counter. J Nucl Med. 2007;48:1197-1206.

20. Tantawy MN, Peterson TE. Simplified $\left[{ }^{18} \mathrm{~F}\right] \mathrm{FDG}$ image-derived input function using the left ventricle, liver, and one venous blood sample. Mol Imaging. 2010;9:76-86

21. Cheng JC, Shoghi K, Laforest R. Quantitative accuracy of MAP reconstruction for dynamic PET imaging in small animals. Med Phys. 2012;39:1029-1041.

22. Sidhu JS, Rajawat YS, Rami TG, et al. Transgenic mouse model of ventricular preexcitation and atrioventricular reentrant tachycardia induced by an AMP-activated protein kinase loss-of-function mutation responsible for WolffParkinson-White syndrome. Circulation. 2005;111:21-29.

23. Rabbani N, Godfrey L, Xue M, et al. Glycation of LDL by methylglyoxal increases arterial atherogenicity: a possible contributor to increased risk of cardiovascular disease in diabetes. Diabetes. 2011;60:1973-1980.

24. Fessler JA, Rogers L. Spatial resolution properties of penalized-likelihood image reconstruction: space invariant tomographs. IEEE Trans Image Process. 1996;5: 1346-1358.

25. Thomas AJ, DaSilva JN, Lortie M, et al. PET of $(R)-{ }^{11} \mathrm{C}$-rolipram binding to phosphodiesterase-4 is reproducible and sensitive to increased norepinephrine in the rat heart. J Nucl Med. 2011;52:263-269.
26. Klein R, Renaud JM, Ziadi MC, et al. Intra- and inter-operator repeatability of myocardial blood flow and myocardial flow reserve measurements using rubidium-82 pet and a highly automated analysis program. J Nucl Cardiol. 2010; 17:600-616.

27. Lamoureux M, Thorn SL, Dumouchel T, et al. Uniformity and reproducibility of normal resting myocardial blood flow in rats with ${ }^{13} \mathrm{~N}$-ammonia and small animal PET. Nucl Med Commun. 2012;33:917-925.

28. Simões MV, Egert S, Ziegler S, et al. Delayed response of insulin-stimulated fluorine-18 deoxyglucose uptake in glucose transporter-4-null mice hearts. J Am Coll Cardiol. 2004;43:1690-1697.

29. Cook GJ, Lodge MA, Marsden PK, Dynes A, Fogelman I. Non-invasive assessment of skeletal kinetics using fluorine-18 fluoride positron emission tomography: evaluation of image and population-derived arterial input functions. Eur J Nucl Med. 1999;26:1424-1429.

30. Anselm DD, Anselm AH, Renaud J, et al. Altered myocardial glucose utilization and the reverse mismatch pattern on rubidium-82 perfusion/F-18-FDG PET during the sub-acute phase following reperfusion of acute anterior myocardial infarction. J Nucl Cardiol. 2011;18:657-667.

31. Birnie D, de Kemp RA, Tang AS, et al. Reduced septal glucose metabolism predicts response to cardiac resynchronization therapy. J Nucl Cardiol. 2012;19:73-83.

32. Mielniczuk LM, Birnie D, Ziadi MC, et al. Relation between right ventricular function and increased right ventricular $\left[{ }^{18} \mathrm{~F}\right]$ fluorodeoxyglucose accumulation in patients with heart failure. Circ Cardiovasc Imaging. 2011;4:59-66.

33. vom Dahl J, Herman WH, Hicks RJ, et al. Myocardial glucose uptake in patients with insulin-dependent diabetes mellitus assessed quantitatively by dynamic positron emission tomography. Circulation. 1993;88:395-404. 